\title{
(Des)igualdades de género, avances normativos y políticas de igualdad en la Universidad Nacional de Río Negro (Patagonia, Argentina)
}

\section{Resumen}

Durante 2017, la Universidad Nacional de Río Negro (UNRN) de la Patagonia, Argentina incorporó profundos cambios a nivel normativo con implicancias en la promoción de la igualdad de género entre las que se destacan la aprobación de un "Protocolo para Actuación sobre Violencias de Género" así como también los ejes principales para diseñar en el corto plazo y de un modo participativo un "Plan de Igualdad" que promueva políticas de equidad para reducir las desigualdades existentes entre varones y mujeres. Todas estas reformas no hubieran sido posible sin el impulso y la lucha de las mujeres de la universidad de todos los claustros.
Este artículo tiene como objetivo dar cuenta de los cambios institucionales que la agenda feminista está produciendo en la UNRN, de la importancia de vincular las investigaciones aplicadas con las políticas públicas de la universidad, de las obligaciones internacionales que tiene la UNRN en esta materia, así como los desafíos pendientes para que las nuevas políticas se traduzcan en una reducción significativa de las desigualdades que padecemos las mujeres en todos los ámbitos.

Palabras clave: Universidad, Género, Políticas de Igualdad, Feminismo.

POR MARIANA RULLI Doctora en Ciencias Sociales (Facultad Latinoamericana de Ciencias Sociales-FLACSO), Politóloga (Universidad de Buenos Aires-UBA). Profesora e Investigadora del Centro Interdisciplinario de Estudios sobre Derechos, Inclusión y Sociedad (CIEDIS) de la Universidad Nacional de Río Negro (UNRN), Argentina. mrulli@unrn.edu.ar 


\section{Abstract}

During 2017, the National University of Río Negro (UNRN) of Patagonia, Argentina incorporated profound changes at the normative level with implications in the promotion of gender equality, among which the approval of a "Protocol for Action on Gender Violence" stands out. as well as the main axes to design in the short term and in a participatory way an "Equality Plan" that promotes equity policies to reduce existing inequalities between men and women. All these reforms would not have been possible without the impulse and struggle of the women of the university of all the cloisters.

This article aims to account for the institutional changes that the feminist agenda is producing in the UNRN, the importance of linking the applied research with the public policies of the university, the international obligations that the UNRN has in this matter, as well as the as the pending challenges so that the new policies translate into a significant reduction in the inequalities that women suffer in all areas.

Keywords: University, Gender, Equality Policies, Feminism.

\section{Resumo}

Durante 2017, a Universidade Nacional do Río Negro (UNRN) da Patagonia, Argentina incorporou profundas mudanças no nível normativo com implicâncias na promoção da igualdade de gênero entre as que se destacam a aprovação de um "Protocolo para Atuação sobre Violências de Gênero" assim como também os eixos principais para desenhar no corto prazo e de um modo participativo um "Plano de Igualdade" que promova políticas de equidade para reduzir as desigualdades existentes entre varões e mulheres. Todas estas reformas não tivessem sido possíveis sem o impulso e a luta das mulheres da universidade de todos os claustros.

Este artigo tem como objetivo dar conta das mudanças institucionais que a agenda feminista está produzindo na UNRN, da importância de vincular as pesquisas aplicadas com as políticas públicas da universidade, das obrigações internacionais que a UNRN tem nesta materia, assim como os desafios pendentes para que as novas políticas se traduzam em uma redução significativa das desigualdades que padecemos as mulheres em todos os âmbitos.

Palavras chave: Universidade, Gênero, Políticas de Igualdade, Feminismo.

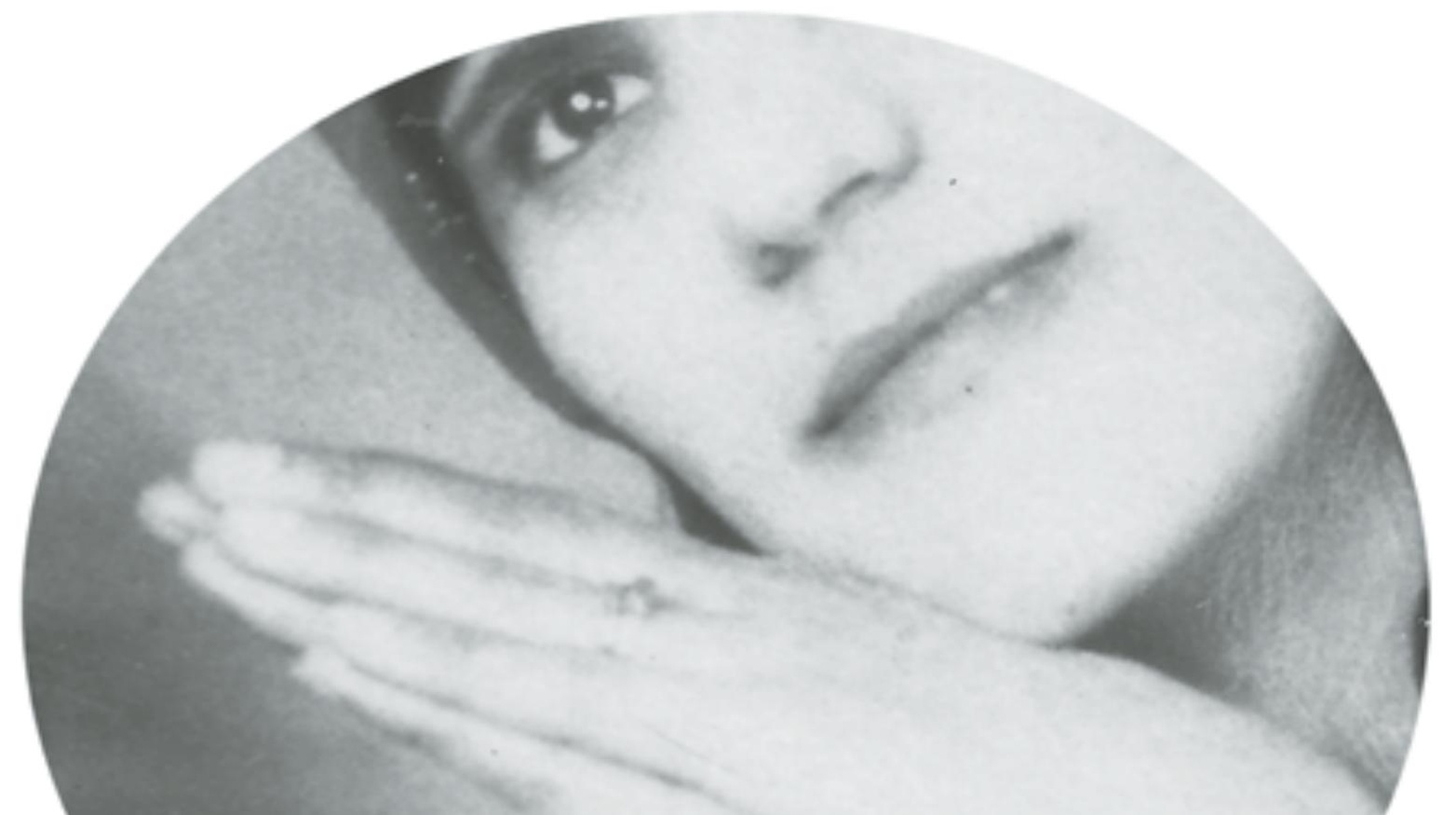




\section{Introducción}

Las desigualdades de género, que están cimentadas sobre la base del modelo patriarcal y androcéntrico, atraviesan todos los espacios de nuestras sociedades. Las mujeres sufrimos esta desigualdad en la distribución de ingresos, en el acceso y desempeño en el mercado laboral, en el acceso a la justicia y a la seguridad social, en la participación política, en el ámbito doméstico y (mal llamado) privado, entre otros.

El escenario de la educación superior no es diferente. En la región, según la Organización Universitaria Interamericana (OUI), el 14\% de los rectores de las universidades latinoamericanas son mujeres. En el caso de Argentina: de todas las universidades e institutos universitarios que componen el Consejo Interuniversitario Nacional (CIN) el 89\% de los rectores son varones, sólo 6 rectoras de 55 instituciones. Tampoco la Universidad Nacional de Río Negro (UNRN) es una excepción a un cuadro general de desigualdad de género (Rulli, Peter y Robles, 2016).

La desigualdad en la participación de las mujeres de los procesos de toma de decisiones en todos los niveles de gobierno y de instituciones de la sociedad civil, incluso en las universidades, es una de las deudas pendientes de la transición a la democracia en la región que imposibilitan forjar una agenda feminista y transformadora, que constituya el marco para el debate y diseño de políticas públicas que desafíen y ataquen a las desigualdades existentes y consecuentemente, a favor de la erradicación de cualquier forma de violencia y discriminación por género.

Durante 2017, la Universidad Nacional de Río Negro (UNRN) de la Patagonia, Argentina incorporó profundos cambios a nivel normativo con implicancias en la promoción de la igualdad de género. En junio, se aprobó un "Protocolo para Actuación sobre Violencias de Género", así como también los ejes principales para diseñar en el corto plazo y de un modo participativo un "Plan de Igualdad" que promueva políticas de equidad para reducir las desigualdades existentes entre varones y mujeres dentro de la universidad. En noviembre y a través de la Asamblea Universitaria, se reformó el Estatuto de la UNRN incluyendo modificaciones fundamentales en términos de igualdad de género, como el uso del lenguaje no sexista en todo el articulado del Estatuto. Se estableció que no podrán trabajar en la universidad aquellos que hayan sido sentenciados en causas por violencia de género, equiparando esta situación al caso de los condenados por ser autores o cómplices de delitos de lesa humanidad. Asimismo, se instituyó que las listas para los órganos colegiados de gobierno deberán integrarse en proporciones paritarias de varones y mujeres y se adoptó el criterio de representación igualitaria de género en la composición de los jurados de concursos docentes, acordando además que quienes resulten acusados por violencia directa e indirecta de género no podrán ser parte de jurados o tribunales.

\section{La desigualdad en la participación de las mujeres de los procesos de toma de decisiones en todos los niveles de gobierno y de instituciones de la sociedad civil, incluso en las universidades, es una de las deudas pendientes de la transición a la democracia en la región...}

Todas estas reformas no hubieran sido posible sin el impulso y la lucha de las mujeres de la universidad de todos sus claustros. Este artículo tiene como objetivo dar cuenta de los cambios institucionales que la agenda feminista está produciendo en la UNRN, de la importancia de vincular las investigaciones aplicadas con las políticas públicas de la universidad, de las obligaciones internacionales que tiene la UNRN en esta materia, así como los desafíos pendientes para que las nuevas políticas se traduzcan en una reducción significativa de la desigualdad que padecemos las mujeres en todos los ámbitos. Para ello, se presentan los antecedentes normativos nacionales e internacionales como la creación de la Red Interuniversitaria por la Igualdad y contra las Violencias de Género en Argentina y el escenario de la educación superior nacional. También, se analizan datos sobre la desigualdad de género en los espacios de 
participación política y cargos jerárquicos de la UNRN. Luego, se exponen los antecedentes al proceso de debate y sanción de un "Protocolo de Actuación contra las Violencias de Género" y de los ejes para la elaboración de un "Plan de Igualdad" en la UNRN. Finalmente, se presentan reflexiones acerca de los desafíos próximos vinculados a la implementación del Protocolo y la elaboración del Plan.

\section{Antecedentes y marco normativo internacional, regional y nacional}

Según la Convención sobre la Eliminación de todas las formas de Discriminación contra las Mujeres (CEDAW $)^{1}$ la "discriminación contra la mujer" es: "toda distinción, exclusión o restricción basada en el sexo que tenga por objeto o por resultado menoscabar o anular el reconocimiento, goce o ejercicio por la mujer, independientemente de su estado civil, sobre la base de la igualdad del hombre y la mujer, de los derechos humanos y las libertades fundamentales en las esferas política, económica, social, cultural y civil o en cualquier otra esfera", (CEDAW, Art. 1).

La CEDAW obliga a los Estados Partes a tomar "todas las medidas apropiadas, incluso de carácter legislativo, para asegurar el pleno desarrollo y adelanto de la mujer, con el objeto de garantizarle el ejercicio y el goce de los derechos humanos y las libertades fundamentales en igualdad de condiciones con el hombre", estableciendo, también, el principio de debida diligencia estatal, que resulta fundamental para garantizar a las mujeres el goce efectivo de sus derechos (CEDAW, Art. 3).

Recientemente, en 2015, el examen periódico de la CEDAW dijo sobre Argentina: "A pesar de los logros en términos de equidad de género en el ámbito educativo y de los innegables avances en el mercado de trabajo y en la participación femenina en emprendimientos económicos, en general las mujeres argentinas siguen enfrentando segregación vertical y horizontal en el ámbito laboral. Su inserción en el mercado de trabajo no trajo aparejada una incorporación masiva de los hombres al mundo del cuidado y las tareas domésticas, produciendo fenómenos como el de la doble jornada laboral como lo demuestra la Encuesta del Uso del Tiempo del año 2013". Y agregaba que: “es ampliamente mayor la presencia de mujeres en el ámbito universitario: en las universidades públicas la inscripción de mujeres alcanzó en 2011, el 57.5\% del total de estudiantes y $56.3 \%$ de las privadas, elevándose esos números al $61.5 \%$ y $62.1 \%$ entre los graduados, respectivamente".

En ese contexto, Argentina adhirió en 2015 a la nueva agenda de Objetivos de Desarrollo Sostenible (ODS): se comprometió a eliminar para el 2030 cualquier forma de discriminación contra las mujeres y niñas. Anteriormente, en el 2000, Argentina había incluido como una de las metas nacionales para el logro de los Objetivos de Desarrollo del Milenio (ODM): "Alcanzar en el año 2015 una mayor equidad de género mediante una mejor participación económica de la mujer y la reducción de la brecha salarial entre varones y mujeres, manteniendo los niveles de igualdad de géneros alcanzados hasta el año 2000 en el ámbito educativo", logrando para 2015 una reducción de la brecha salarial al $20 \%$.

En noviembre de 2016 la Relatora Especial de Naciones Unidas sobre la Violencia contra las mujeres realizó una misión en Argentina. En su informe presentado al Consejo de Derechos Humanos en 2017, señaló que la violencia contra la mujer tiene sus raíces en las desigualdades y la discriminación de que son objeto. Para combatirlas, especialmente en el ámbito universitario, recomendó introducir, junto con los programas de estudios de género, una educación específica acerca de la Convención sobre la eliminación de todas las formas de discriminación contra la Mujer (CEDAW), las recomendaciones generales del Comité de la CEDAW y su jurisprudencia, la Convención de Belém do Pará $^{2}$ y la jurisprudencia pertinente de la Corte Interamericana de Derechos Humanos.

En 2017, en el informe del Grupo de Trabajo sobre la Cuestión de la Discriminación contra la Mujer en la Legislación y en la Práctica de la ONU se resaltó la importancia de la efectiva asignación de recursos para apoyar la observancia progresiva de los derechos humanos de la mujer como obligación de los Estados. Específicamente, señala que "los Estados deben emprender un proceso de presupuestación con perspectiva de género para asegurar que sus compromisos jurídicos y normativos arrojen resultados. Entre los principales factores que limitan las buenas prácticas determinadas, 


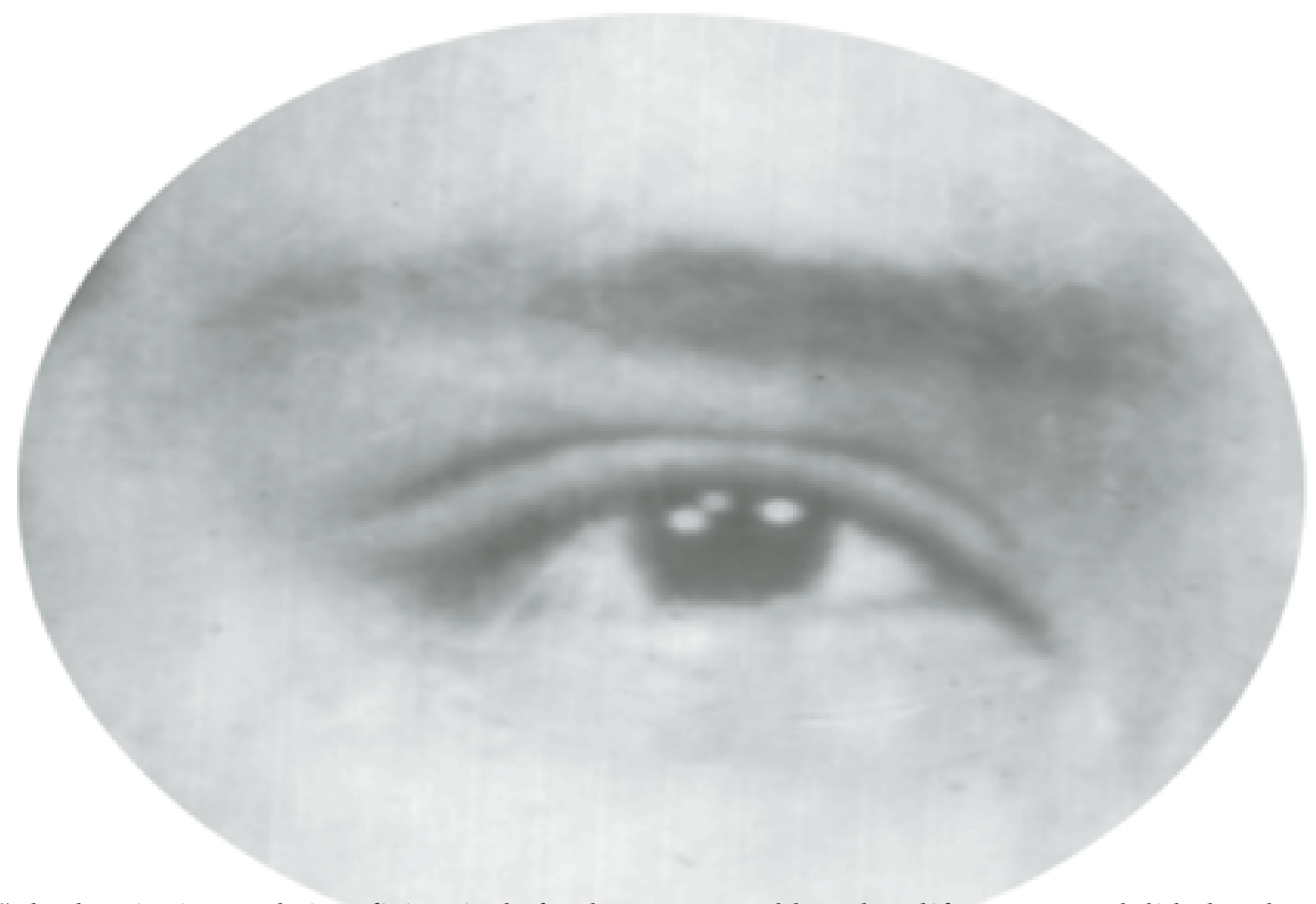

cabe señalar los siguientes: la insuficiencia de fondos, la carga desproporcionada impuesta a los actores no gubernamentales en relación con la aplicación, y la dependencia, en materia de financiación con cargo a recursos internacionales, de un único donante o de la financiación a gran escala".

En el plano nacional, la Ley 26.485/2010 conocida como la ley de "Protección Integral para Prevenir, Sancionar y Erradicar la Violencia Contra las Mujeres” define a la violencia de género o contra la mujer como "toda conducta, acción u omisión, disposición, criterio o práctica discriminatoria que de manera directa o indirecta, tanto en el ámbito público como en el privado, basada en una relación desigual de poder, afecte su vida, libertad, dignidad, integridad física, psicológica, sexual, económica o patrimonial, como así también su seguridad personal o que ponga a la mujer en desventaja con respecto al varón. Se considera violencia indirecta, a los efectos de la presente ley, toda conducta, acción, omisión, disposición, criterio o práctica discriminatoria que ponga a la mujer en desventaja con respecto al varón”. Esta ley nacional, a la que la UNRN debe adecuarse, contempla como tipos de violencia la física, psicológica, sexual, económica y patrimonial, y establece las diferentes modalidades de violencia abarcando la doméstica, institucional, laboral, contra la libertad reproductiva, obstétrica y mediática (véase artículo 3 y 4).

En el escenario de la Educación Superior, según la Red Interuniversitaria por la Igualdad de Género y contra las Violencias ${ }^{3}$, dieciocho universidades nacionales argentinas han aprobado Protocolos de Actuación contra la Violencia de Género y doce universidades se encuentran en proceso de elaboración y/o aprobación. Algunos de estos protocolos se inscriben en programas más amplios para abordar las violencias basadas en el género, como la Universidad de San Martín y la Universidad Nacional de Rosario, por ejemplo. Sin embargo, no hay antecedentes de elaboración de planes de igualdad en las universidades argentinas como el que acaba de aprobar la UNRN.

En el escenario universitario internacional, las universidades españolas cuentan con Planes de Igualdad, en América Latina, la Universidad Nacional Autónoma de México y la Pontificia Universidad Católica del Perú, por ejemplo. También otras norteamericanas, como Yale, y europeas, como Oxford y Libre de Berlín cuentan con programas de género. 


\section{Las desigualdades de género en las universidades argentinas}

En el Gráfico 1 se presenta la distribución de funciones y de la jerarquía institucional en las universidades argentinas. A partir de los datos, se puede observar la segregación horizontal ${ }^{4}$ y vertical ${ }^{5}$ en la distribución de las funciones y autoridades.

\section{El caso de la Universidad Nacional de Río Negro (Patagonia, Argentina)}

Desde 2015, en un contexto de movilización y activismo feminista a nivel nacional, en la UNRN comenzó un proceso impulsado por mujeres que promovió cambios institucionales. Este proceso puede dividirse en tres etapas: la primera etapa "político-electoral" coincide con las elecciones universitarias en 2015; la segunda "político-académica" es a partir de la presentación de informes y artículos que analizaron las desigualdades de género en la universidad en 2016; la tercera etapa es la "político-participativa" en la que mujeres de la universidad discutieron y elaboraron una propuesta de Protocolo de Actuación contra las Violencias y la propuesta de un Plan de Igualdad. En las subsecciones siguientes se presentan y analizan las mencionadas etapas.

\section{La etapa político-electoral: las elecciones de 2015 en la UNRN}

En 2015 una lista ${ }^{6}$ de candidatas/os a los Consejos Directivos $^{7}$ de una de las sedes de la UNRN ${ }^{8}$ fue impugnada por la lista oficialista y obligada, por la Junta Electoral de la universidad, a readecuar los lugares de sus candidatas/os por tener más mujeres que varones. Esta impugnación fue realizada basándose en la Ley de cupo femenino ${ }^{9}$ que establecía que las listas de candidatos/as para el Congreso Nacional debía estar compuesta por el $30 \%$ de mujeres en lugares expectables de ser electas. La utilización de la Ley de Cupo Femenino y su reglamentación, no para fomentar y garantizar los derechos participativos de las mujeres sino, precisamente, para cercenarlos, fue de una interpretación tan errónea como única. En ese momento, la Junta Electoral de la UNRN consideró a los varones

Gráfico 1. Distribución de autoridades universitarias, Argentina (2016).

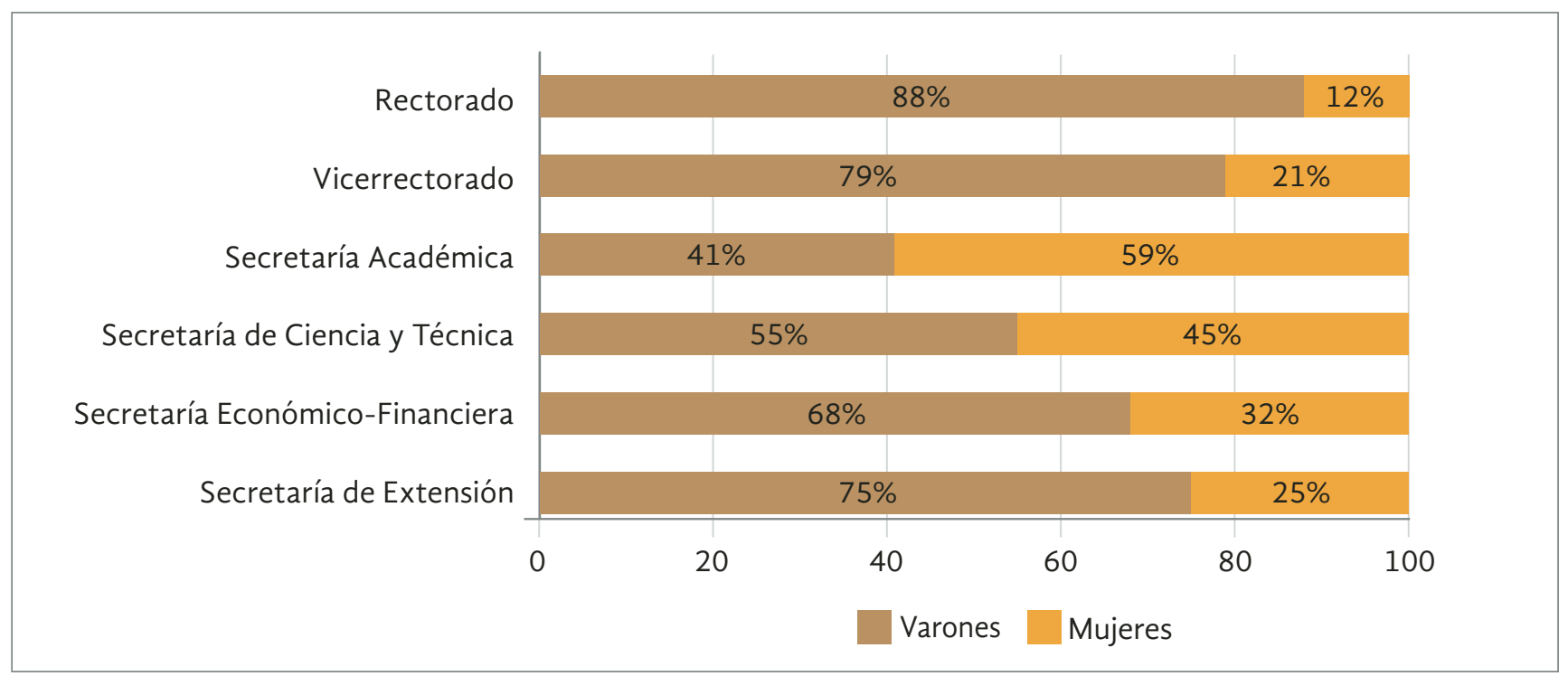


Cuadro 1. Población universitaria, según género. (Comparación entre universidades nacionales y la UNRN).

\begin{tabular}{|ccccc}
\hline Población & \multicolumn{2}{c}{ UUNN } & \multicolumn{2}{c}{ UNRN } \\
& Mujeres & Varones & Mujeres & Varones \\
\hline Estudiantes & $58 \%$ & $42 \%$ & $60 \%$ & $40 \%$ \\
\hline Ingresantes & $58 \%$ & $42 \%$ & $57 \%$ & $43 \%$ \\
\hline Egresados/as & $61 \%$ & $39 \%$ & $62 \%$ & $38 \%$ \\
\hline Docentes & $49 \%$ & $51 \%$ & $51 \%$ & $49 \%$ \\
\hline Profesores/as & $43 \%$ & $57 \%$ & $46 \%$ & $54 \%$ \\
\hline Auxiliares & $52 \%$ & $48 \%$ & $54 \%$ & $46 \%$ \\
\hline
\end{tabular}

Fuente: OAC y Anuario Estadístico 2014- SPU.

discriminados, en contra de cualquier dato de la realidad y de los compromisos jurídicos contraídos por el Estado Nacional en esta materia como las exigencias de la Comisión Interamericana de Derechos Humanos, ${ }^{10}$ así como tratados internacionales en la materia ${ }^{11}$ y la vasta literatura académica sobre el tema ${ }^{12}$.

En definitiva, la exigencia impuesta por la Junta Electoral fundada en que la lista de Espacio Plural tenía demasiadas mujeres fue violatoria de las normas internacionales y nacionales vigentes, desconociendo la dimensión histórica de la lucha por los derechos de las mujeres, permitiendo la consolidación y perpetuación de la situación de discriminación de la participación de las mujeres en nuestra universidad.

\section{La etapa político-académica:}

las desigualdades en la UNRN

A partir de lo sucedido en las elecciones de 2015, el 8 de marzo de 2016, un grupo de mujeres investigadoras realizaron y presentaron un informe que analiza datos cuantitativos sobre las desigualdades de género existentes en la UNRN en cuanto a la participación política y también en las dificultades de ascensos en la carrera académica por la segregación vertical y horizontal. El escenario de desigualdad descripto no era muy diferente al de cualquier otra universidad argentina (ni al que presenta la mayoría de las universidades en todo el mundo).

En los datos sobre la población global según género de la UNRN se pueden observar los mismos parámetros en comparación con el resto de las universidades nacionales argentinas. En los datos presentados en el Cuadro 1, se visualiza que salvo en el caso de los profesores, en todas las otras categorías de población universitaria hay un mayor porcentaje de mujeres.

Sin embargo, la mayor presencia de mujeres en toda la población universitaria no se ve reflejada en los cargos de gestión ni jerarquía. Actualmente, el rector y los tres vicerrectores de la UNRN son varones. Desde la creación de la universidad en 2009, sólo una vez hubo una vicerrectora en la Sede Andina. En el Gráfico 2 se presentan los datos de la distribución según género de las autoridades de la universidad. Como se observa, únicamente en el caso de las secretarias del rectorado hay paridad, en las restantes autoridades el porcentaje de varones es mayor que el de mujeres.

En el caso de las direcciones de las carreras (licenciaturas, ciclos de complementación y tecnicaturas), mientras en las sedes Andina y Alto Valle y Valle Medio hay un escenario de paridad, en la Atlántica de once direcciones una está ocupada por una mujer (Cuadro 2).

Cuadro 2. Directores/as de carrera, según género (por sede, 2018).

\begin{tabular}{|ccc|}
\hline Sede & Varones & Mujeres \\
\hline Andina & 9 & 10 \\
\hline Alto Valle y Valle Medio & 11 & 10 \\
\hline Atlántica & 10 & 1 \\
\hline
\end{tabular}

Fuente: Elaboración propia en base a www.unrn.edu.ar 
Gráfico 2. Autoridades de la UNRN, según género (2018).

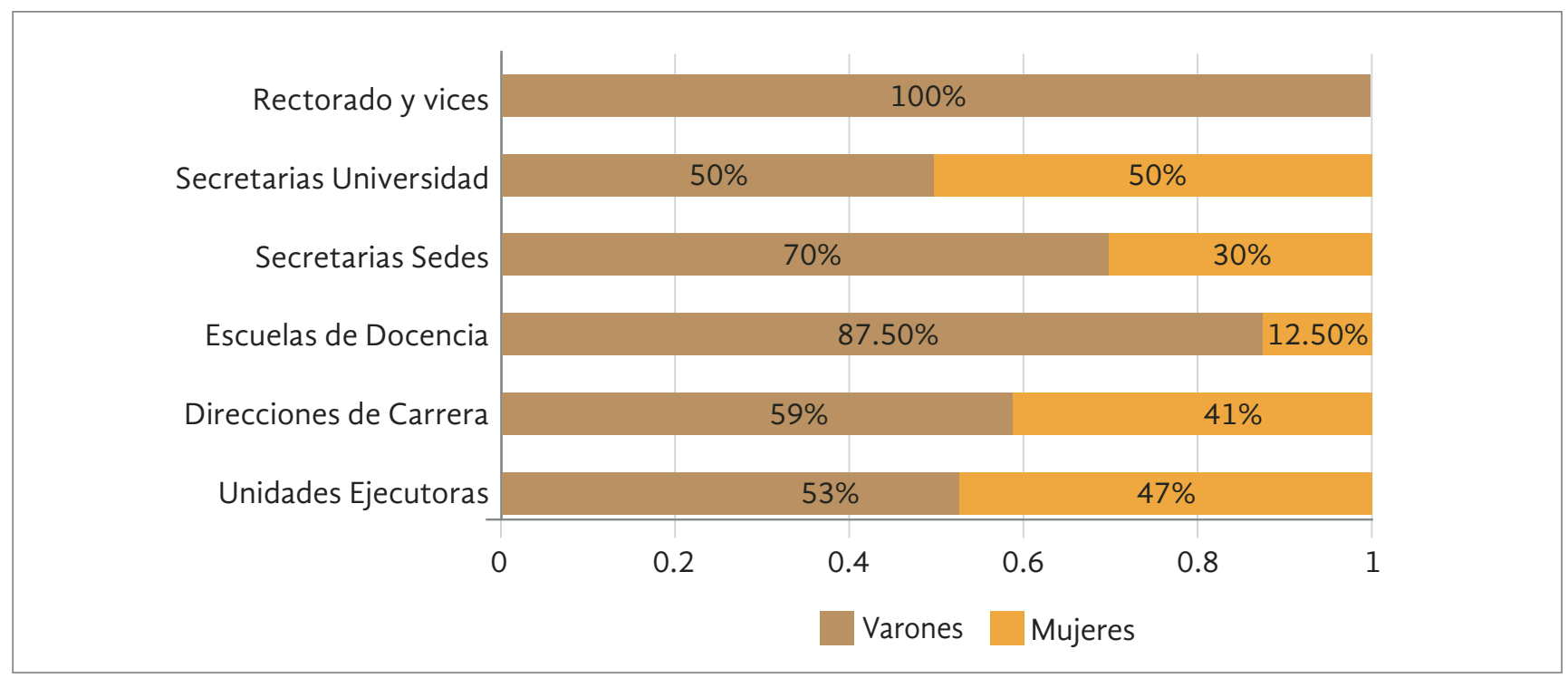

Fuente: Elaboración propia en base a www.unrn.edu.ar

Cuadro 3. Directores/as de las unidades ejecutoras, según género (2018).

\begin{tabular}{|ccc|}
\hline UE & Varones & Mujeres \\
\hline Institutos & 3 & 1 \\
\hline Centros & 2 & 3 \\
\hline Laboratorios & 3 & 2 \\
\hline Hospitales Escuela & 1 & 2 \\
\hline Totales UE & 9 & 8 \\
\hline
\end{tabular}

Fuente: Elaboración propia en base a www.unrn.edu.ar

Tal como se observa en el Cuadro 3, en el caso de las unidades ejecutoras de investigación: los centros y laboratorios presentan un escenario casi de paridad en sus autoridades, mientras que en el caso de los institutos se observan tres varones de cuatro en total.

Tal como se mencionó anteriormente, la UNRN cuenta con tres consejos de gobierno: el Consejo de Programación y Gestión Estratégica con el 68\% de varones y el 32\% de mujeres y los Consejos de Investigación, Creación Artística, Desarrollo y Transferencia de Tecnología y de Docencia, Extensión y Vida Estudiantil el $67 \%$ de varones y $33 \%$ de mujeres (Cuadro 4 ).

Esto significa que en la UNRN la reglamentación electoral funcionó como un techo para el ingreso de mujeres y no como un piso mínimo. La promoción del ingreso de las mujeres en los cuerpos colegiados se realizaba a través de la incorporación de cupos o cuotas en la reglamentación electoral. En este sentido, el sistema electoral tiene un importante efecto en la distribución de cargos entre géneros. Incluso a pesar de la literatura que ya reconocía el impacto de las leyes de cupo como techo para las mujeres (Archenti y Tula, 2008, 2011; Caminotti y Cogiano, 2017; Caminotti, 2008; Gadano y Rulli, 2016), la Junta Electoral plasmó una interpretación rígida de la legislación en perjuicio de las posibilidades y oportunidades de que más mujeres resultaran electas en 2015.

\section{Etapa político-participativa: el proceso de debate y sanción del Protocolo y el Plan de Igualdad}

En 2017, a partir de un Encuentro de Mujeres de la UNRN, el 8 de marzo en el que participaron docentes, no docentes y estudiantes, se propuso la elaboración de un Protocolo y un Plan que encuadre políticas integrales que promuevan la igualdad.

El 27 de marzo, las autoridades de la universidad presentaron un proyecto de Protocolo en el Consejo 
de Gestión y Programación Estratégica. La propuesta fue discutida y cuestionada por docentes de la Sede Atlántica que elaboraron dos documentos ${ }^{13}$ que planteaban la necesidad de debatir cuestiones técnicas del protocolo pero, especialmente, la necesidad de enmarcar el Protocolo de actuación en un Plan de Igualdad. Finalmente, el proyecto no se votó y volvió a Comisión planteando la posibilidad de presentar alternativas superadoras.

Con el objetivo de elaborar una propuesta alternativa y superadora al proyecto de las autoridades de la universidad, se realizaron varias reuniones entre mujeres (más de cinco con la participación de mujeres de todos los claustros y sedes). El consenso principal al que se arribó en las primeras reuniones fue el de elaborar conjuntamente la propuesta alternativa del Protocolo y el Plan de Igualdad.

La propuesta de Plan de Igualdad tenía como objetivo general la promoción, el fortalecimiento y el desarrollo de la igualdad de trato y oportunidades entre mujeres y varones. Esto implica la necesidad de realizar un diagnóstico para elaborar objetivos y ejes de trabajo que atraviesen las áreas y decisiones políticas de la institución. Cabe destacar que un Protocolo es el conjunto de procedimientos específicos para actuar en el caso que se detecte violencias de género (es decir, podría haber distintos protocolos para actuar ante diferentes situaciones).
Para la discusión de la propuesta alternativa se ejecutaron diversas acciones: (i) se analizaron varios Planes de Igualdad de universidades de otros países y se sistematizaron sus políticas principales y ejes de actuación; (ii) se analizaron protocolos de actuación contra las violencias de género de otras universidades argentinas; (iii) se elaboró un cuadro comparativo entre el protocolo presentado por las autoridades de la UNRN y el protocolo alternativo preparado por las mujeres que participaron del proceso. También, con el objetivo de intercambiar opiniones y sugerencias con mujeres de otras sedes se realizaron reuniones virtuales y se creó un grupo de Facebook con más de 70 integrantes.

Finalmente, en junio, una vez elaborada una propuesta alternativa, se discutió y consensuó en una mesa de trabajo con las autoridades de la universidad que luego impulsaron su tratamiento y aprobación en el Consejo Universitario. Fue un proceso participativo, de debate y construcción de consenso inédito. Junto con la presentación de la propuesta se inició una campaña de adhesiones a través de un formulario web en el que fueron registradas 497 adhesiones de miembros de la UNRN y de externos y de diferentes instituciones de la sociedad civil como sindicatos. En noviembre, mediante la Asamblea Universitaria se reformó el Estatuto de la UNRN incorporando cambios primordiales en términos de igualdad de género que fueron detallados anteriormente.

Cuadro 4. Consejos de la UNRN, según género (2015-2019).

\begin{tabular}{|ccccccc|}
\hline Consejos & \multicolumn{2}{c}{ CPyGE } & \multicolumn{2}{c}{ CICADyT } & \multicolumn{2}{c|}{ CDEyVE } \\
\hline Claustro/Género & $\mathrm{V}$ & $\mathrm{M}$ & $\mathrm{V}$ & $\mathrm{M}$ & $\mathrm{V}$ & $\mathrm{M}$ \\
\hline Autoridades & 4 & 0 & 4 & 0 & 4 & 0 \\
\hline Profesores/as & 6 & 3 & 6 & 3 & 6 & 3 \\
\hline Auxiliares & 2 & 1 & 2 & 1 & 2 & 1 \\
\hline Estudiantes & 1 & 2 & 1 & 3 & 3 & 1 \\
\hline No docentes & 2 & 1 & 2 & 1 & 1 & 2 \\
\hline Totales & 15 & 7 & 15 & 8 & 15 & 8 \\
\hline Totales en $\%$ & 68 & 32 & 67 & 33 & 67 & 33 \\
\hline
\end{tabular}

Fuente: Elaboración propia en base a www.unrn.edu.ar 


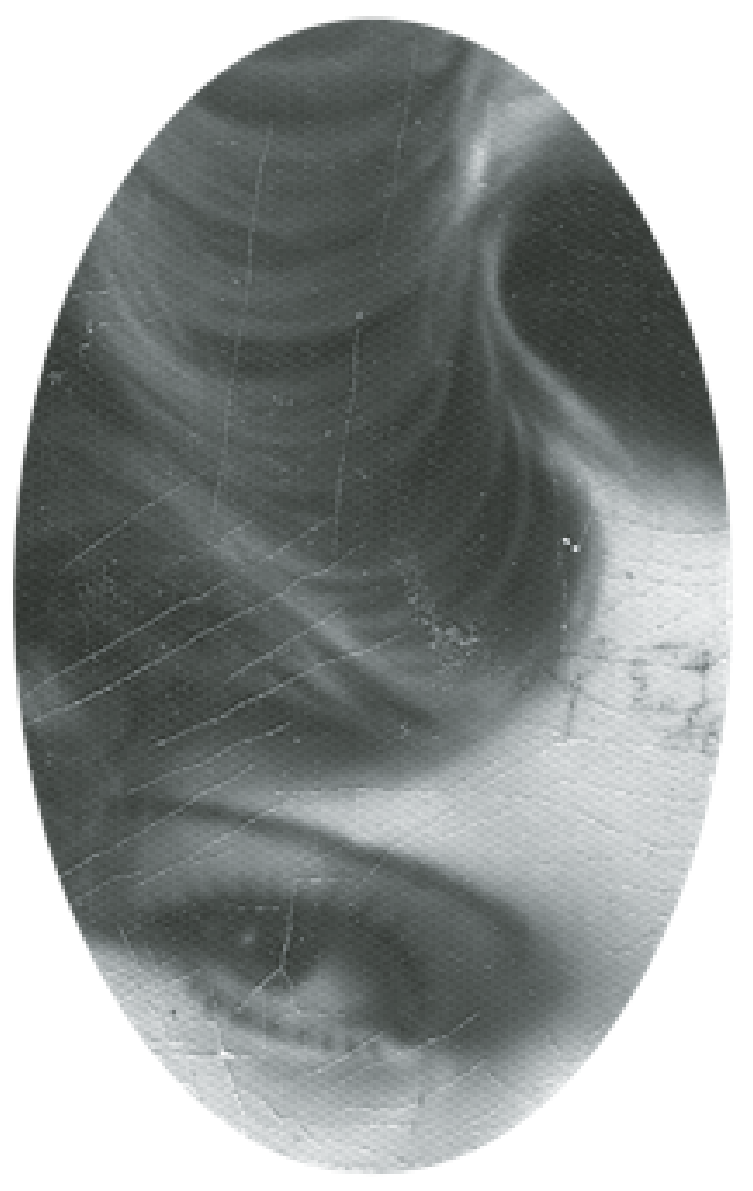

\section{Reflexiones finales}

El camino hacia la eliminación de las desigualdades de género implica una combinación de políticas, cambios en las normativas y especialmente, cambios culturales en todos los ámbitos de la sociedad. Mayor democracia implica igualdad de participación y eso es especialmente relevante en términos de género.

La visibilización de la desigualdad de género en la universidad primero y la aprobación de las políticas mencionadas después ubica a la UNRN en un grupo reducido de universidades nacionales e internacionales que reconocen, al menos en su normativa, de manera explícita el problema de la discriminación contra las mujeres y establece mecanismos y políticas para combatirla. A decir verdad, la UNRN no hace otra cosa que cumplir con los compromisos internacionales que ha asumido el Estado nacional e implementar algunas de las recomendaciones de la CEDAW, la Relatora Especial de Naciones Unidas sobre la Violencia contra las Mujeres y otras iniciativas internacionales $\mathrm{y}$ nacionales que fueron presentadas en la segunda sección de este artículo.

Cada una de las etapas del proceso de incorporación de estas reformas con perspectiva de género fue impulsada desde abajo, por las mujeres universitarias que, a través de procesos de participación, promovieron la creación y ampliación de una agenda feminista en la UNRN.

El proceso de elaboración de un Plan de Igualdad con base en los ejes recientemente aprobados deberá ser participativo y fruto del debate interclaustro, intersedes e interdisciplinar y acompañado por un fuerte compromiso político que incluya la disponibilidad de recursos económicos y personal capacitado en la implementación. La elaboración de un Plan de Igualdad orientado hacia la transformación de las prácticas y cultura que dificultan el desarrollo y la participación plena de las mujeres en la vida institucional, así como de un protocolo que permita efectivamente sancionar las diversas formas de violencia que derivan de las desigualdades de género, son elementos indispensables en la conformación de un programa político de vanguardia. 


\section{Notas}

1. La CEDAW fue adoptada por la Asamblea General de las Naciones Unidas en su Resolución 34/180, de 18 de diciembre de 1979 como resultado de más de 30 años de trabajo de la Comisión de la Condición Jurídica y Social de la Mujer. La Cedaw fue ratificada por nuestro país a través de la Ley Nacional No 23.179, de 1985.

2. Para más información sobre la Convencion de Belém do Pará véase http://www.oas.org/es/mesecvi/convencion.asp

3. La Red fue creada antes de NUM y supo capitalizar las movilizaciones de mujeres que permitieron traccionar dentro de las universidades los debates y políticas en relación a la problemática de género. Las mujeres vamos generando transformaciones profundas de la cultura universitaria a través de la aprobación de los protocolos contra las violencias o las disputas por la ampliación del presupuesto (Vazquez Laba, 2017).

4. La segregación horizontal es cuando las mujeres son concentradas en determinados sectores y lugares de trabajo (docencia, cuidados, servicios domésticos, tareas administrativas) mientras los varones se concentran en otros (construcción, seguridad). Esto implica la feminización y masculinización de las distintas actividades.

5. La segregación vertical se refiere a la baja proporción de las mujeres en los cargos jerárquicos y directivos o en los sectores con mayor reconocimiento profesional.

6. La lista impugnada fue denominada Lista Espacio Plural.

7. La UNRN cuenta con tres órganos colegiados de gobierno a nivel universidad: el Consejo de Programación y Gestión Estratégica, el Consejo de Investigación, Creación Artística, Desarrollo y Transferencia de Tecnología y el Consejo de Docencia, Extensión y Vida Estudiantil. Los mismos consejos están presentes en cada una de las sedes académicas. La asamblea universitaria está conformada por los tres consejos y la persona a cargo del rectorado (Véase Estatuto de la UNRN disponible en linea en https://www.unrn.edu.ar/images/ Estatuto_UNRN_-_Vigente_diciembre_2014.pdf

\section{Bibliografía}

Archenti, N. y M. I. Tula, (2011) “Ciudadanía y representación. A 20 años de la Ley de Cuotas de Género” en Revista Sociedad, núm. 29-30.

Archenti, N. y M. I. Tula, (2014) "Cambios normativos y equidad de género. De las cuotas a la paridad en América Latina. Los casos de Bolivia y Ecuador" en América Latina Hoy. Revista de Ciencias Sociales, (66): 47-68.

(eds). (2008) Mujeres y política en América Latina. Sistemas electorales y cuotas de género, Heliasta, Buenos Aires.

Caminotti, M. y N. Cogliano (2017) "La paridad política en Argentina: avances y desafíos", Programa Naciones Unidas para el Desarro1lo/ONU-Mujeres/IDEA Internacional, Buenos Aires.

(2008) "Derribar los muros indebidos. Reflexiones en torno de las leyes de cupo femenino en Argentina. Aportes para el Estado y la Administración Gubernamental. Editorial”, Asociación de Administradores Gubernamentales, Buenos Aires; p. 13.

Cánaves, V. (2011) Participación política de las mujeres y acceso a espacios de decisión. Algunos argumentos de sentencias judiciales para recuperar y reflexionar, Equipo Latinoamericano de Justicia y Género, [PDF]
8. La UNRN tiene tres sedes académicas en distintas ciudades de la provincia de Río Negro: La Sede Andina que se encuentra ubicada en Bariloche y El Bolsón; la Sede Atlántica en Viedma y San Antonio Oeste y la Sede Alto Valle que se encuentra ubicada en las ciudades de General Roca, Cipolletti y Villa Regina y Choele Choel. En este artículo se ha tomado como muestra los datos de la Sede Atlántica. La impugnación fue en la Sede Atlántica.

9. La Ley de Cupo Femenino (Ley Nacional Nro. 24.012) fue sancionada en Argentina en pionera en el mundo. Recientemente, en 2017 fue modificada y actualmente las listas deben estar confeccionadas con una participación equivalente de género. Para más información sobre la Ley de Cupo Femenino en Argentina véase Archenti y Tula (2011).

10. Caso $\mathrm{N}^{\mathrm{o}} 11.307$.

11. Pacto Internacional de Derechos Civiles y Políticos, Art. 25; Convención Americana de Derechos Humanos, Arts. 23.1; Convención para la Eliminación de Todas las Formas de Discriminación contra la Mujer, Art. 7; Declaración Universal de Derechos Humanos, Art. 21 , entre otros.

12. Ver los documentos de trabajo y experiencias comparadas de "ONU Mujeres" en http://www.unwomen.org/esde Y más específicamente Cánaves, Violeta, "Participación política de las mujeres y acceso a espacios de decisión. Algunos argumentos de sentencias judiciales para recuperar y reflexionar", Equipo Latinoamericano de Justicia y Género, 2011.

13 La Línea de Género, Trabajos y Diversidad Familiar del Centro Interdisciplinar de Estudios sobre Derechos Inclusión y Sociedad (CIEDIS) elaboró un documento que analizaba la propuesta oficial y también la agrupación política Espacio Plural.
Gadano, L. y M. Rulli (2016a) "La importancia de la paridad de género en los espacios de decisión” en Diario Río Negro, 14 de octubre.

Rulli, M. et al. (2016) (Des)Igualdad de Género en la Universidad Nacional de Río Negro. Aportes para el Debate, trabajo presentado en el XII Congreso Nacional y V Congreso Internacional sobre Democracia, organizado por la Facultad de Ciencia Política y Relaciones Internacionales de la Universidad Nacional de Rosario. Rosario, 12 al 15 de septiembre.

Vázquez Laba, V.y C. Rugna (2015) "La lucha es en el campus, el claustro y el pasillo" en Revista Anfibia, Noviembre 2015. Disponible en: http://www.revistaanfibia.com/la-lucha-es-en-el-campus-elclaustro-y-el-pasillo/

Tula, M. I. Mujeres y Política. (2015). "Mujeres y política. Un panorama sobre adopción de las cuotas de género y sus efectos en América Latina y Colombia" en Revista Ópera, Bogotá, pp. 9-33. 

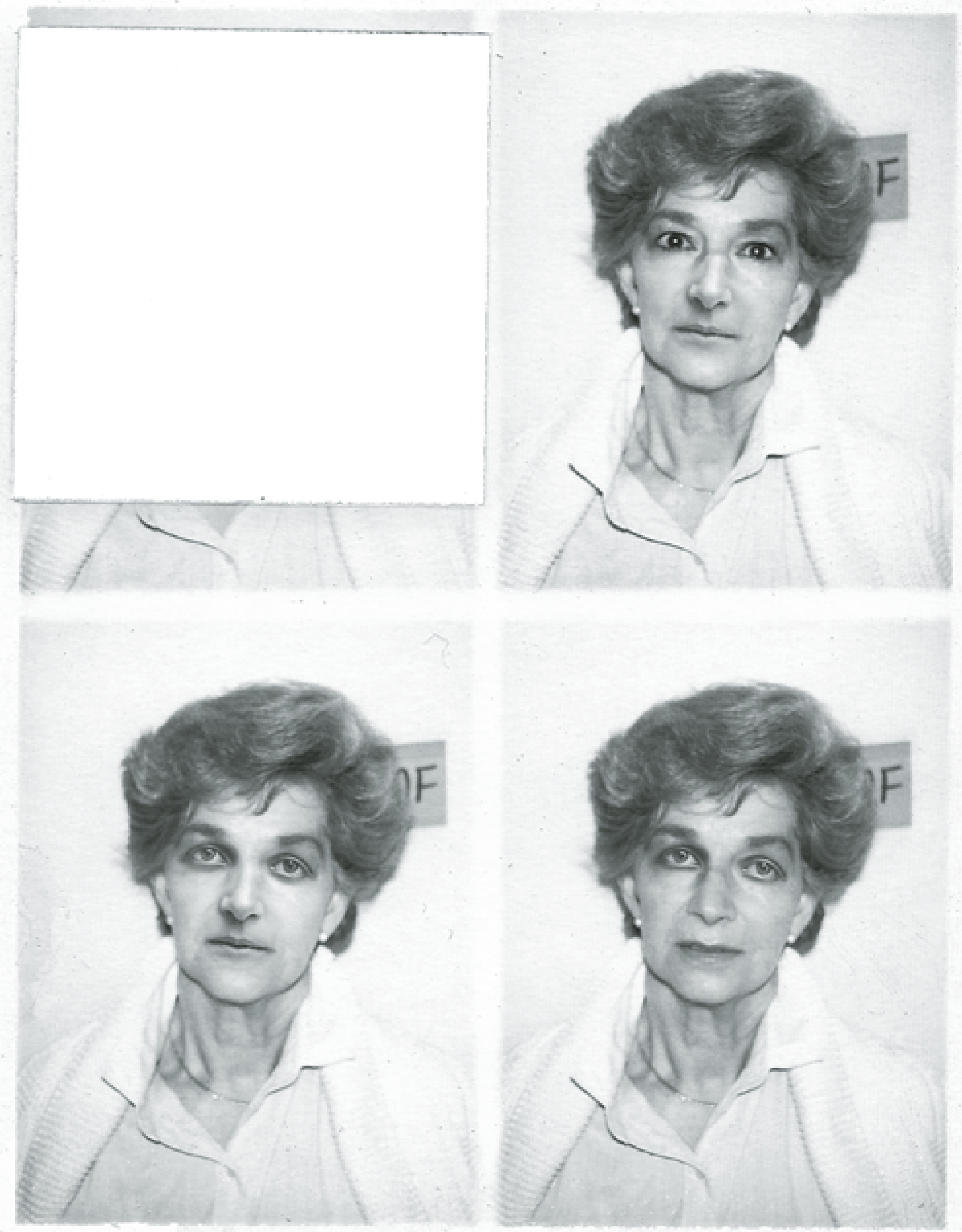